\title{
Relevance of long term time - series of atmospheric parameters at a mountain observatory to models for climate change
}

\author{
Mária Kancírová \\ Košice, Slovakia \\ Faculty of Science, P. J. Šafárik University, Šrobárova 2, 04108 Košice, Slovakia \\ E-mail: maria.kancirova@vsbm.sk

\section{Karel Kudela ${ }^{1}$} \\ Institute of Experimental Physics SAS, Watsonova 47, 04001 Košice, Slovakia \\ E-mail: kkudela@saske.sk
}

Department of Mathematics and Physics, The University of Security Management, Kukučinova 17, 04001

\section{Anatoly D. Erlykin}

Department of Physics, Durham University, South Road, Durham, DH1 3LE, UK

Permanent address: P.N. Lebedev Physical Institute of the Russian Academy of Sciences, 53 Leninskij

Prospekt, 119991, Moscow, Russia

E-mail: a.d.erlykin@durham.ac.uk

\section{Arnold W. Wolfendale}

Department of Physics, Durham University, South Road, Durham, DH1 3LE, UK

E-mail: a.w.wolfendale@durham.ac.uk

A detailed analysis has been made of annual meteorological, and cosmic ray, data from the Lomnicky stit mountain observatory (2634 masl), from the standpoint of looking for possible solar cycle (including cosmic ray) manifestations.

Apart from the Pressure, Sunspot number correlation, we find no significant Solar Cycle dependence (i.e. difference from one Cycle to the next) of the relationship between local temperature and pressure, cloud cover and pressure, cloud cover and Sunspot Number, and cloud cover and cosmic rays, corrected for atmospheric pressure, or not, in contrast with the results of others for the cloud cover, cosmic ray intensity correlation. The apparent rise in cloud cover with increasing cosmic ray intensity is smaller than found by others and is, in our view, due at least in part to atmospheric pressure effects.

The 34th International Cosmic Ray Conference

30 July- 6 August, 2015

The Hague, The Netherlands

\section{${ }^{1}$ Speaker}




\section{Introduction}

The Earth's climate has always been changing. The reasons for these changes, however, have always been subject to discussions and in need of further analysis. The case of the contribution of cosmic rays (CR) to the magnitude of cloud cover (CC) is an example. Although the likelihood of its being very small is high, e.g. the IPCC report [1] it is not necessarily zero.

Authors in [2] find that the solar influence on cloud cover is not uniquely defined by one solar driver (cosmic rays and the UV irradiance), but both seem to play a role depending on the climatic conditions and altitude.

Paper [3] identified a significant correlation between CR and CC. At first sight the correlation appeared to have a natural explanation in terms of the role of $\mathrm{CR}$ ions forming cloud - condensation nuclei $(\mathrm{CCN})$ upon which cloud droplets preferentially form. However, later work in some detail, e.g. [4, 5, 6], showed that the effect, if it existed at all, was much smaller than claimed. Nevertheless, there do appear effects to be explained and it is possible that CR have an indirect effect on $\mathrm{CC}$, for example by way of the atmospheric electric circuit $[7,8,9]$. Thus further study is needed.

In this article we examine the atmospheric parameters form the Lomnicky stit (LS) mountain observatory [10]. Because CR are dependent on the Solar Cycle (SC) we examine the two SC (SC22 and SC23) separately.

\section{The basic data}

The manner in which the data are collected is discussed elsewhere [11] and only a summary will be given here, of relevance to those parameters involved in the present analysis.

These are: Cloud cover - 'cloudiness' (CC) [12], Atmospheric pressure (P), Atmospheric temperature (T) [13], Cosmic ray intensity from neutron monitor data: CRCORR (corrected for atmospheric pressure) and CRUNCORR (uncorrected).

\section{The analysis}

\subsection{Pressure, temperature and cloud cover}

In Figure 1, we plot of the annual averages of the pressure cover against two different meteorological parameter; namely temperature for 30 years (1984 - 2013) and cloud cover for 32 complete years covered by the cloud data 1982 - 2013 and separately for SC22 (1986 - 1996) and SC23 (1996 - 2008). The relationship between atmospheric pressure and cloud cover has a well known meteorological origin. When a low-pressure area moves in, the air rises, cools and condenses out moisture, which forms clouds and precipitation. The correlation coefficient between these two parameters and pressure is shows Table 1 .

\begin{tabular}{|l|c|c|c|c|c|c|}
\hline \multirow{2}{*}{} & \multicolumn{2}{|c|}{$1982-2013$} & \multicolumn{2}{c|}{ SC22 } & \multicolumn{2}{c|}{ SC23 } \\
\cline { 2 - 7 } & $\mathrm{T}$ & LSCC & $\mathrm{T}$ & LSCC & $\mathrm{T}$ & LSCC \\
\hline $\mathrm{P}$ & 0.52 & -0.63 & 0.90 & -0.47 & 0.82 & -0.42 \\
\hline
\end{tabular}

Table 1 Linear correlation coefficient between pressure and temperature and cloud cover at Lomnicky stit. 

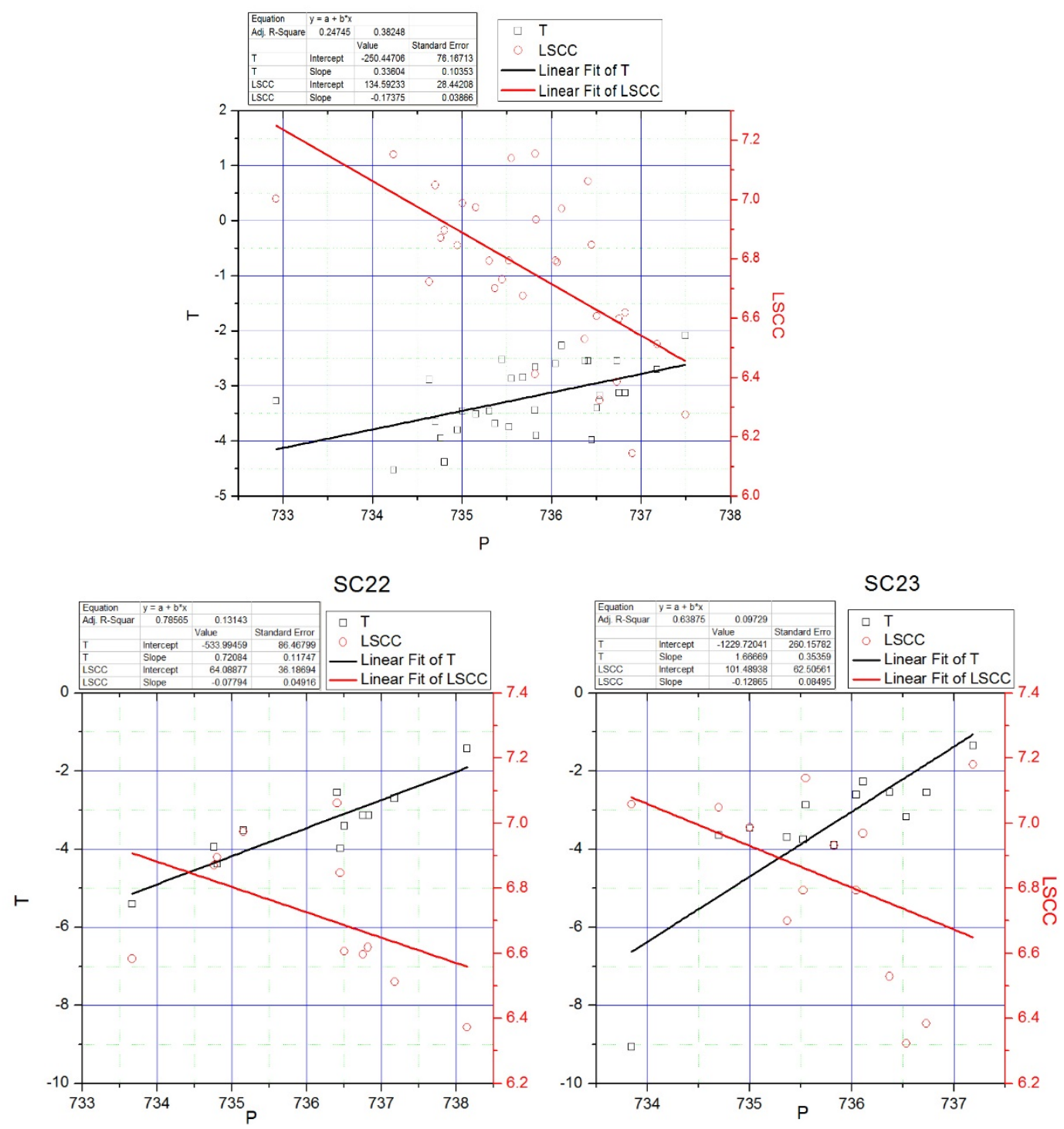

Figure 1 Temperature and cloud cover versus pressure (annual means) for the whole period (upper panel) and for separate cycles 22 and 23 (lower panels). The whole period $(1982-2013)$ is somewhat longer than that of SC22 plus SC23.

3.2 Sunspot number (SSN), pressure and cloud cover

Figure 2 shows dependence between sunspot number and pressure and cloud cover at Lomnicky stit. Table 2 shows the results.It is clear that there is a weak correlation.

\begin{tabular}{|l|c|c|c|c|c|c|}
\hline \multirow{2}{*}{} & \multicolumn{2}{|c|}{$1982-2013$} & \multicolumn{2}{c|}{ SC22 } & \multicolumn{2}{c|}{ SC23 } \\
\cline { 2 - 7 } & $\mathrm{P}$ & LSCC & $\mathrm{P}$ & LSCC & $\mathrm{P}$ & LSCC \\
\hline SSN & 0.47 & -0.26 & 0.36 & -0.15 & 0.04 & -0.06 \\
\hline
\end{tabular}

Table 2 Linear correlation coefficient between sunspot number and pressure and cloud cover at Lomnicky stit.

The correlation may be related to that in the CR intensity, as discussed in the next section. 

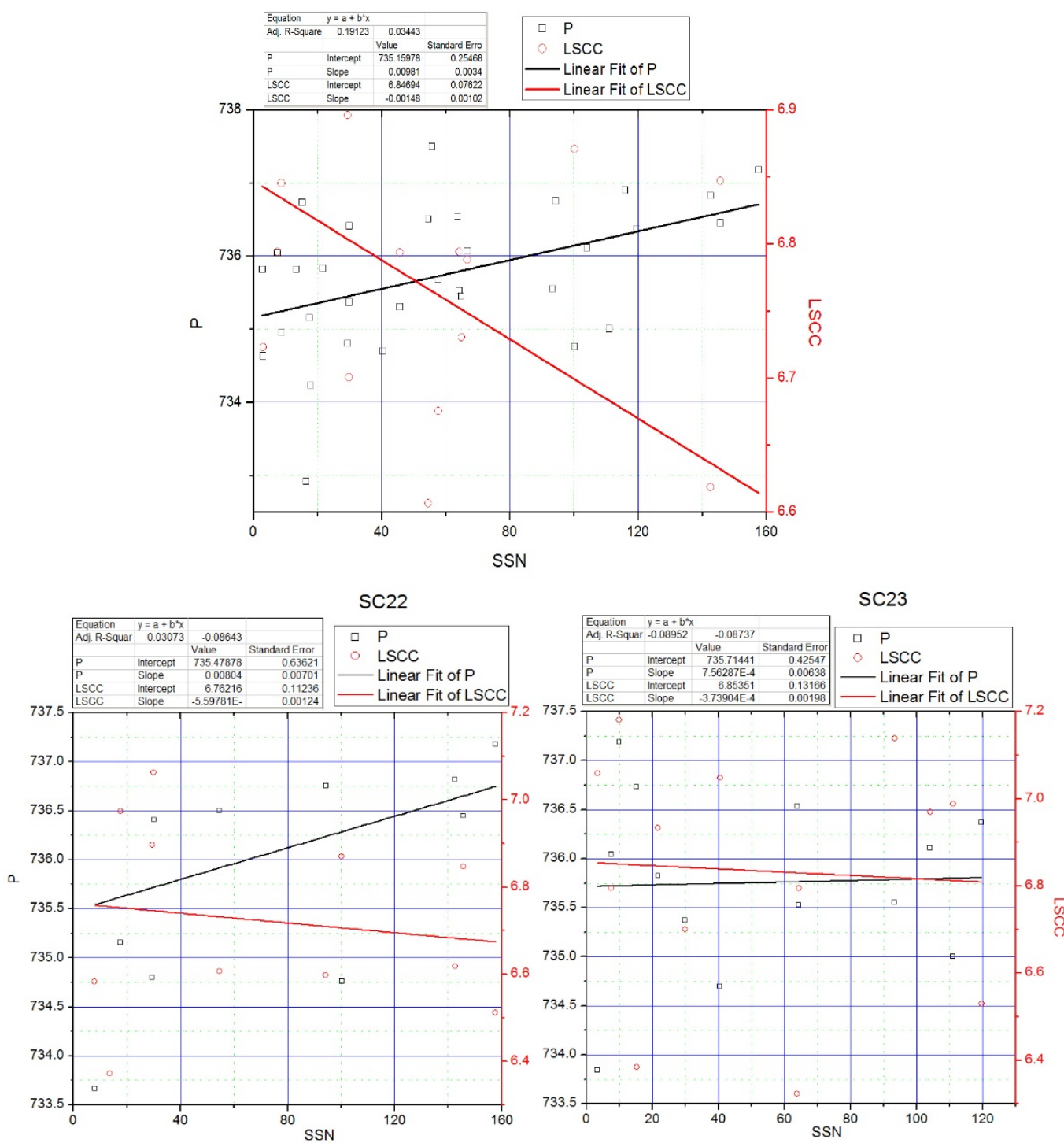

Figure 2 Cloud coverage and pressure versus sunspot number (annual means) for the whole period studied (upper panel) and for solar cycles 22 and 23 (lower panels). The whole period is somewhat longer than that of SC22 plus SC23 and the total number (32) is correspondingly greater than $11+13$.

\subsection{Cosmic ray intensity and cloud cover}

Figure 3 shows the straight forward correlation of LSCC and CR (corrected and uncorrected).

\begin{tabular}{|l|c|c|c|c|c|c|}
\hline & \multicolumn{2}{|c|}{$1982-2013$} & \multicolumn{2}{c|}{ SC22 } & \multicolumn{2}{c|}{ SC23 } \\
\hline & CRCORR & CRUNCORR & CRCORR & CRUNCORR & CRCORR & CRUNCORR \\
\hline LSCC & 0.27 & 0.32 & 0.10 & 0.13 & 0.42 & 0.47 \\
\hline
\end{tabular}

Table 3 Linear correlation coefficient between cosmic ray intensity (corrected and uncorrected) and cloud cover at Lomnicky stit. 
It will be noted that the values for the correlation are at the 1.93 and 2.3 standard deviations levels, i.e. bordering on significant.
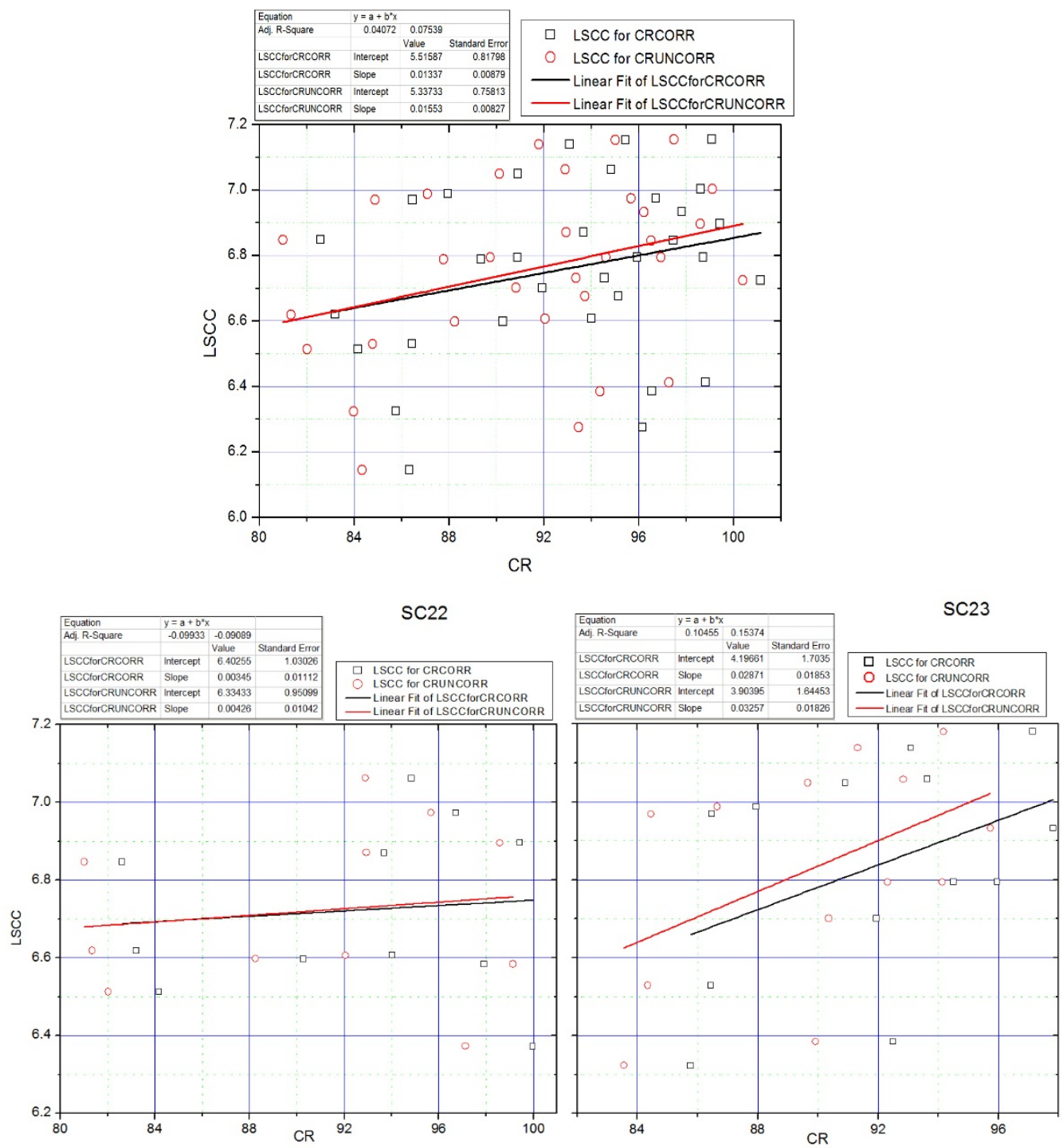

Figure 3 Cloud cover versus corrected and uncorrected CR intensity at LS (annual means) for the whole period (upper panel) and for separate cycles 22 and 23 (lower panels). The whole period is somewhat longer than that of SC22 plus SC23 and the total number (32) is correspondingly greater than $11+13$.

\section{Summary}

Concerning the CR correlations, the lack of a difference between the correlations for corrected and uncorrected CR intensities (annual means) points against the hypothesis that $\mathrm{CR}$ ionization is responsible for significant $\mathrm{CC}$ changes.

As remarked already (Introduction) a correlation need not be causal and we consider this to be the cause for the CC, $\mathrm{CR}$ ( $\mathrm{SSN})$ correlations.

The results are thus in favour of those reported in introduction which disfavour the ionization hypothesis. 
Previous work using subsets of the present mountain data [14] found 'slightly different' correlation coefficients for averaging periods ranging from 1 day via 10 days to 100 days and longer periods, but an overall value is only marginally different from zero.

\section{Acknowledgments}

The authors are grateful to the Kohn Foundation for financial support. KK wishes to acknowledge VEGA grant agency project 2/0040/13 for support.

\section{References}

[1] IPCC Fifth Assessment Report. 2014.

[2] M. Voiculescu, I. G. Usoskin, K. Mursula, Different response of cloud to solar input, Geophys. Res. Let. 33, 21 (2006), DOI: 10.1029/2006GL027820.

[3] H. Svensmark, Cosmoclimatology: a new theory emerges. News and Reviews in Astronomy and Geophysics, 48, 1 (2007), doi: 10.1111/j.1468-4004.2007.48118.x.

[4] A. D. Erlykin, G. Gyalai, K. Kudela, T. Sloan, A. W. Wolfendale, Cosmic Rays and Global Warming. Paper 1.15, Proceedings of ECRS 2008, September, Kosice, Solvakia.

[5] A. D. Erlykin, G. Gyalai, K. Kudela, T. Sloan, A.W. Wolfendale, Some aspects of ionization and the cloud cover, cosmic ray correlation problem. Journal of Atmospheric and Solar-Terrestrial Physics. 71, 8-9 (2009), doi:10.1016/j.jastp.2009.03.007.

[6] A. D. Erlykin, T. Sloan, A. W. Wolfendale, Correlations of clouds, cosmic rays and solar irradiation over the Earth. Journal of Atmospheric and Solar-Terrestrial Physics. 72, 2-3 (2010), doi:10.1016/j.jastp.2009.11.002.

[7] R. G. Harrison, M. H. P. Ambaum, Enhancement of cloud formation by droplet charging. Proceedings of the Royal Society A. 2008, DOI: 10.1098/rspa.2008.0009.

[8] K. L. Aplin, R. G. Harrison, M. J. Rycroft, Investigating Earth's atmospheric electricity: a role model for planetary studies. Space Science Reviews, 137, 1-4, pp. 11-27, 2008, ISSN 0038-6308. DOI: $10.1007 / \mathrm{s} 11214-008-9372-\mathrm{x}$.

[9] B. A. Tinsley, R. P. Rohrbaugh, M. Hei, Electro-scavenging in clouds with broad droplet size distributions and weak electrification. Atmospheric Research. 59-60, 2001, doi:10.1016/S01698095(01)00112-0.

[10] D. Židek, P. Lipina, Instruction for the observers of meteorological stations (in Czech). Metodický předpis No 13. Czech Hydrometeorological Institute, Ostrava, p. 90, 2003.

[11] International Satellite Cloud - Climatology Project (ISCCP). [online]. http://isccp.giss.nasa.gov. (access Feb. 27, 2012).

[12] Cloud data obtained from Slovak Hydrometeorological Institute (SHMU).

[13] National Climatic Data Center - NOAA. [online]. http://www.ncdc.noaa.gov/ (accessed Jan. $30,2014)$

[14] M. Kancírová, K. Kudela, Cloud cover and cosmic ray variations at Lomnický štít high altitude observing site. Atmospheric Research. 149, pp. 166-173, 2014, doi:10.1016/j.atmosres.2014.06.004. 